Cahiers $d u$ MONDE RUSSE

\section{Cahiers du monde russe}

Russie - Empire russe - Union soviétique et États indépendants

$49 / 4 \mid 2008$

Destins individuels et terreur. Jeunesse dans la société post-stalinienne

\title{
Kathryn Hendley, ed., Remaking the Role of Law
}

\section{Aurore Chaigneau}

\section{(2) OpenEdition}

Journals

Édition électronique

URL : https://journals.openedition.org/monderusse/6981

DOI : 10.4000/monderusse. 6981

ISSN : $1777-5388$

Éditeur

Éditions de l'EHESS

Édition imprimée

Date de publication : 28 décembre 2008

Pagination : 800-803

ISBN : 978-2-7132-2197-2

ISSN : $1252-6576$

Référence électronique

Aurore Chaigneau, "Kathryn Hendley, ed., Remaking the Role of Law », Cahiers du monde russe [En ligne], 49/4 | 2008, mis en ligne le 24 décembre 2009, consulté le 03 septembre 2022. URL : http:// journals.openedition.org/monderusse/6981; DOI : https://doi.org/10.4000/monderusse.6981

Ce document a été généré automatiquement le 3 septembre 2022

Tous droits réservés 


\title{
Kathryn Hendley, ed., Remaking the Role of Law
}

\author{
Aurore Chaigneau
}

\section{RÉFÉRENCE}

Kathryn HENDLEY, ed., Remaking the Role of Law. Commercial Law in Russia and the CIS. Huntington, NY: Juris publishing, 2007, 315 p.

1 Quels sont le rôle et l'usage du droit dans les transformations institutionnelles récentes? Tel peut être l'angle par lequel on s'intéresse à l'ouvrage collectif dirigé par Kathryn Hendley et consacré aux réformes de la sphère commerciale en Russie et dans la CEI. Élaboré à partir d'un colloque organisé en 2004 sur les réformes juridiques dans la CEI, le présent recueil rassemble des contributions d'éminents spécialistes anglo-saxons et russes sur l'évolution du droit, essentiellement en Russie, dont certaines ont été ajoutées ou réactualisées pour la publication en 2007.

2 Il est structuré autour de trois axes principaux : politique, analyse juridique, sociologie du droit, qui servent d'entrée dans le droit commercial. On les retrouve à travers un découpage en quatre parties. La première traite des enjeux politiques inhérents à la réforme du droit commercial, la deuxième des fondements du droit commercial et de son évolution. La troisième partie aborde la question de la mise en œuvre pratique de certaines réformes clés et enfin la quatrième lui fait écho à travers le thème plus spécifique de l'impact des réformes sur l'investissement étranger.

Analyse politique des réformes: Le propos s'ouvre sur une analyse par Peter Solomon des tentations actuelles de contre-réforme judiciaire. La révision des règles de l'organisation judiciaire, posées entre 1990 et 2000, ainsi que les tentatives de réformer certains mécanismes institutionnels sont le signe d'un changement d'époque et de rupture avec la mandature de Boris El'cin. Sous la présidence de Putin, les assauts contre l'institution n'ont cessé de se multiplier à l'initiative tantôt du Parlement, tantôt de l'administration 
présidentielle. Les dysfonctionnements de cette administration sont autant de prétextes pour remettre en cause des principes fondamentaux: pour exemple, la tentative de Sergej Mironov (au Conseil de la Fédération) de modifier la composition et les attributions de la Chambre de qualification judiciaire (Kvalifikacionnaja kollegija). Cet organe, créé en 1989, est chargé de statuer sur la nomination des magistrats et les avancements de carrière. Mironov a tout essayé pour recomposer ces chambres et imposer la présence de "juristes» non magistrats désignés par le corps législatif. Ces propositions auraient certainement vu le jour si les juristes les plus puissants du Parlement et les juges eux-mêmes n'étaient sortis de leurs gonds pour défendre l'institution en 2004. Il est en outre suggéré que le programme de rénovation de la justice du ministère du Développement économique a servi à amadouer les magistrats en prônant une amélioration substantielle de leurs conditions de travail. Depuis le printemps 2000, ce ministère, dirigé par German Gref, est en effet chargé de mettre en place un programme de rénovation de la justice. Ce dernier a par ricochet ranimé les débats sur la justice et conduit corrélativement à une remise en cause des principes fondamentaux de l'organisation judiciaire comme l'inamovibilité des magistrats. L'idée de contrats à durée déterminée a circulé dans les couloirs. Dans la même veine, il a été envisagé que la Cour constitutionnelle ne rende plus que des avis consultatifs dépourvus de toute portée obligatoire, ou encore qu'elle soit délocalisée à Saint-Pétersbourg. En matière de réforme, les esprits s'avèrent fertiles et les propositions toujours plus imaginatives pour contourner les premiers effets de l'état de droit (contourner les jurés au pénal, le recours aux tribunaux militaires, etc.).

4 L'image négative de la magistrature et de la justice dans l'opinion publique offre une large marge de manœuvre au pouvoir. La corruption est toujours brandie comme prétexte à la refonte des statuts, ce qui permet au pouvoir de manier grossièrement la carotte et le bâton avec les juges. À chaque fois, il s'en faut de peu que les plus mauvaises idées ne soient concrétisées, tant il est facile de susciter les critiques à l'égard d'un corps peu populaire. La corruption ne saurait pourtant être l'apanage du monde judiciaire, comme le montre l'article de L. Shelley. L'auteur y décrit le développement parasitaire des liens entre oligarques et bureaucrates concernant le développement du monde des affaires. La perpétuation des pratiques criminelles depuis la période soviétique témoigne de la continuité des personnes et des méthodes. Les ramifications des clans criminels dans les plus hautes sphères de l'État et du monde de l'entreprise, la captation, partielle, de ces activités par le crime organisé sont les témoins de la gangrène du système. Le secteur bancaire notamment est, selon L. Shelley, l'otage d'anciens membres du parti et des services de sécurité qui ont usé de leurs ressources au sein du pouvoir pour sécuriser leurs activités.

5 Ces pratiques se traduisent par quelques phénomènes biens spécifiques qui dessinent le capitalisme à la russe. Des « banques de poches » (pocket bank) sont chargées de la gestion des fonds publics. Elles permettent à leur propriétaire de faire de l'argent avec celui de l'État sans encourir de responsabilité, hors de toute transparence. De même, l'institutionnalisation des kryši (parrains) se généralise dans le monde des affaires. Elle est pointée comme la cause du faible développement des petites et moyennes entreprises. Enfin, les plus grands abus ont entouré la privatisation. La bureaucratie s'est alliée avec les élites économiques pour capter les richesses économiques. On parle même de privatisation de l'État, tant la connivence entre oligarques et hauts fonctionnaires est inextricable. 
6 Analyse juridique: La deuxième partie de l'ouvrage aborde la politique législative. A. L. Makovskii, l'un des corédacteurs du Code civil de 1994, revient sur les transformations historiques de la législation civile et sur les choix opérés lors de la préparation des codes $\mathrm{du} \mathrm{xx}^{\mathrm{e}}$ siècle, comme celui d'un code unique pour le droit civil et commercial. Un second article de A. Dovgert se penche sur le code ukrainien, ses difficultés d'application et l'impact du développement économique sur cette législation nouvelle. O.M. Kozyr, spécialiste de droit immobilier, revient sur le développement récent de ce pan de la législation en Russie, suite à la privatisation des immeubles commerciaux et de logements. Enfin M. Zhiltsov traite du droit international privé russe suite à la réforme du 1er mars 2002 et à l'adoption de la partie IV du Code civil de la Fédération de Russie. L'ensemble ces textes offre un premier aperçu de la tradition juridique russe et de l'intégration de raisonnements en termes d'analyse économique du droit par la doctrine russe.

7 Sociologie du droit: La troisième partie retient tout particulièrement l'attention en ce qu'elle aborde la question de l'impact des réformes législatives à travers divers exemples comme la création d'une haute administration de la concurrence, les réformes de procédure, les avancées en matière de protection de la réputation des personnes morales ou encore l'enregistrement des personnes morales. Cette dernière réforme de $2002 \mathrm{a}$ sensiblement modifié le cadre du développement des PME en Russie. Elle témoigne d'une volonté politique de "débureaucratisation» et de simplification des procédures d'enregistrement par la création d'un « guichet unique ». Cette procédure a certainement contribué à l'accroissement du nombre d'entreprises légalement enregistrées. Elle innove en outre en faisant peser la responsabilité de la véracité des données enregistrées sur l'État. Mais, en pratique, cette réforme a engendré de longues files d'attente, notamment à Moscou, car elle a rendu obligatoire un réenregistrement de toutes les personnes morales avant la fin 2002, ce qui a aggravé l'engorgement des services. L'objectif indirect de ces mesures était de mettre à jour la liste des entreprises soumises aux impôts fédéraux et de rayer des registres les coquilles vides. Mais, pour ce faire, il a finalement fallu recourir à un dispositif ad hoc permettant de rayer les entreprises fantômes par une pure procédure administrative mise en place en 2005. Effet pervers, la simplification des procédures d'enregistrement semble avoir favorisé les «entreprises d'un jour ", créées pour masquer des transactions illégales ou des évasions fiscales, car les autorités n'ont pas le temps de vérifier les documents et les déclarations qui leur sont soumis. Une nouvelle réforme est aujourd'hui réclamée, notamment par le ministère des Affaires intérieures, qui, traditionnellement, s'oppose aux mesures de dérégulation de l'économie.

8 Au stade judiciaire, l'impact de l'évolution de la législation sur les pratiques des entrepreneurs est tout aussi notable. Comme le souligne K. Hendley, Le nouveau Code de procédure d'arbitrage a opéré un certain nombre de changements pour les acteurs économiques. Depuis 1995, l'instauration de délais stricts pour statuer pose des problèmes pratiques pour les affaires difficiles et la bonne administration de la preuve. La création en 2002 d'une audience préalable des parties a fait évoluer le cours du procès (en offrant deux mois supplémentaires) et modifié le rôle du juge, sans résoudre pratiquement tous les problèmes posés par les délais. Malgré tout, les juges continuent d'être présentés comme une garantie d'efficacité et de bon fonctionnement de l'administration. Les auteurs de la réforme espèrent que l'audience préalable permettra au juge d'amener les parties à une sorte de médiation, ce qui est peu vérifié à ce jour. 
Rien n'est prévu en outre pour les parties qui ne se présentent pas aux audiences et en particulier aux audiences préparatoires ${ }^{1}$. C'est pourtant un cas très fréquent qui, selon les enquêtes de terrain, laisse aux juges un sentiment de gâchis et de perte de temps.

9 En définitive, les thèmes épars de l'ouvrage permettent d'entr'apercevoir les changements de pratiques par et autour de certains pans de la législation commerciale. Il faudrait aborder encore bien d'autres sujets pour esquisser le visage réel du droit commercial et du capitalisme russes. On peut regretter par exemple que l'ensemble des articles ne permette pas de reconstituer une opération économique dans sa totalité. Mais telle n'était pas l'ambition des auteurs.

\section{NOTES}

1. Avant chaque audience, le juge doit convoquer une première fois les parties pour recueillir tous les documents et éléments de preuves et s'assurer de leurs prétentions. Cette première rencontre, obligatoire, est appelée audience préparatoire. 